\title{
En el sellado del catéter venoso central en hemodiálisis, ¿presenta el uso de citrato respecto al uso de heparina menos complicaciones de infección? Una revisión de la literatura
}

\author{
Mateu Nadal Servera ${ }^{1,3}$, Ester Sánchez Marimónn ${ }^{2,3}$ \\ ${ }^{1}$ Enfermero. Unidad de Hemodiálisis. Hospital Son Llàtzer, ${ }^{2}$ Enfermera. Unidad de Cuidados Intensivos. Hospital \\ Universitari Son Espases, ${ }^{3}$ Estudiante de Máster Universitario de Investigación en Salud y Calidad de Vida. Palma \\ de Mallorca. España
}

\section{Resumen}

Introducción: La heparina es el fármaco anticoagulante de elección y de uso habitual en los servicios de hemodiálisis, en el procedimiento de permeabilización de los catéteres venosos centrales, aunque su uso no está exento de riesgos como la hemorragia. La infección asociada al catéter, bien por colonización del catéter o por bacteriemia, es el evento más importante y la segunda causa de mortalidad tras la enfermedad cardiovascular en el paciente en hemodiálisis. En este sentido, el uso del citrato se está presentando como una alternativa para el sellado de los catéteres venosos centrales, dado que tiene propiedades tanto anticoagulantes locales como antimicrobianas y podría ser más eficiente en la prevención o la reducción de la infección asociada al catéter.

Objetivos: Identificar qué anticoagulante de uso en el sellado del catéter venoso central, la heparina o el citrato, presenta menos complicaciones de bacteriemia asociada al catéter y de colonización del catéter;

Material y Método: se realizó una búsqueda bibliográfica a través del metabuscador EBSCOhost (Academic Search Premier, CINAHL) y las bases de datos PubMed, IME, Ibecs, LILACS, CUIDEN, Scopus y Cochrane Library, con la selección final de 15 documentos tras la aplicación de los límites de búsqueda, la eliminación de duplicados y la aplicación de los criterios de inclusión/exclusión. Los resultados más relevantes y actua-

\footnotetext{
Correspondencia:

Mateu Nadal Servera

Unidad de Hemodiálisis. Hospital Son Llàtzer.

Ctra. Manacor Km 4. 07198 Palma. Islas Baleares. España

E-mail:mnadals@hsll.es
}

les disponibles en la literatura científica presentaron cierta variabilidad, tanto en las concentraciones de anticoagulante como en la incidencia de infección.

Resultados y Discusión: La numerosa evidencia sobre la bacteriemia asociada al catéter, tanto en relación con la concentración como en la combinación del anticoagulante de sellado, indica que el citrato $4 \%$ podría reducir la incidencia de bacteriemia, aunque el citrato asociado a antimicrobianos es más efectivo en la prevención de ésta respecto a la heparina sódica o el citrato exclusivo.

Conclusiones: Aunque el uso de citrato sódico exclusivo no presenta claras ventajas en las complicaciones de infección asociada al catéter respecto a la heparina sódica, sí parecen demostrados sus beneficios a bajas $(4 \%)$ o moderadas ( $30 \%$ ) concentraciones, y especialmente, al combinarlo con antibióticos. Aún así, la asociación del citrato con antibióticos debe ser estudiada con profundidad por la comorbilidad del paciente, la reincidencia de infección y la resistencia antibiótica.

In the central venous catheter sealing on hemodialysis, does the use of citrate with respect to the use of heparin have less complications of infection? A review of the literature

\section{Abstract}

Introduction: Heparin is the standard anticoagulant drug commonly used in hemodialysis services for the permeabilization of central venous catheters. Although it has risks like bleeding. Catheter-associated infection, either by colonization of the catheter or bacteraemia, 
is the most important event and the second cause of mortality following cardiovascular disease in the hemodialysis patient. In this sense, citrate use is being presented as an alternative for central venous catheter sealing because it has both local and antimicrobial anticoagulant properties and could be more efficient in preventing or reducing catheter-associated infection.

Objectives: To identify which sealing anticoagulant of the central venous catheter, heparin or citrate, presents less complications of catheter-associated bacteremia and colonization of the catheter.

Material and Method: A bibliographic search was performed through the EBSCOhost (Academic Search Premier, CINAHL) and PubMed, IME, Ibecs, LILACS, CUIDEN, Scopus and Cochrane Library databases, with the final selection of 15 documents after the application of search limits, removal of duplicates and the application of the inclusion/exclusion criteria. The most relevant and current results available in the scientific literature showed some variability, both in anticoagulant concentrations and in the incidence of infection.

Results and Discussion: Numerous evidence on catheter-associated bacteremia, both in relation to the concentration and the combination of the sealed anticoagulant, indicates that $4 \%$ citrate could reduce the incidence of bacteremia. However, antimicrobialassociated citrate is more effective in preventing bacteremia than sodium heparin or exclusive citrate.

Conclusions: Although the use of exclusive sodium citrate does not present clear advantages in the complications of catheter-associated infection with sodium heparin, the benefits are shown at low (4\%) or moderate $(30 \%)$ concentrations, especially when combined with antibiotics. Even so, the association of citrate with antibiotics should be studied according to patient comorbidity, recurrence of infection and antibiotic resistance.

\section{Introducción}

En los últimos años, el número de pacientes con insuficiencia renal crónica ha aumentado y aproximadamente el $89 \%$ de éstos inician el tratamiento sustitutivo renal mediante hemodiálisis ${ }^{1}$. El acceso vascular de elección en hemodiálisis es la fístula arteriovenosa (FAV), a pesar de que un importante porcentaje de pacientes, entre el $15-50 \%$, son portadores de catéteres venosos centrales de acceso central y tunelizado, principalmente yugular ${ }^{2-4}$. El uso del catéter venoso central (CVC) está orientado a la espera del acceso vascular tipo FAV, aunque ante dificultades en el estudio vascular puede ser de carácter definitivo ${ }^{4,5}$. Sin embargo, el uso de catéteres venosos centrales requiere medidas de esterilidad, asepsia e higiene, sin estar exento de riesgos, siendo las complicaciones asociadas más frecuentes la infección, la obstrucción y el bajo flujo intradiálisis por permeabilidad reducida del catéter4. De hecho, en la actualidad las recomendaciones norteamericanas y europeas indican limitar el uso de los catéteres para evitar el aumento de la morbimortalidad de los pacientes en hemodiálisis ${ }^{2,3,5}$.

En este sentido, dichas complicaciones suponen un incremento de los costes sanitarios, por ingreso hospitalario, recolocación del catéter o recambio del catéter entre otros, siendo la infección asociada al catéter (IA-CVC), bien por colonización del catéter o por bacteriemia, el evento más importante y la segunda causa de mortalidad tras la enfermedad cardiovascular ${ }^{2,6-8}$. El origen de la infección más frecuente en los CVC es la colonización endoluminal producida por manipulación a través de las conexiones externas, siendo otras causas la colonización extraluminal producida por migración de la flora cutánea a través del trayecto cutáneo de fibrina, la vía hematógena desde otro punto de infección o la contaminación de los líquidos de infusión ${ }^{2}$. El $75 \%$ de las muertes en pacientes en hemodiálisis son causadas por bacteriemia, y es el acceso vascular la principal vía de entrada de ésta, con un riesgo relativo de mortalidad 1,5 veces mayor y un riesgo relativo de infección 7,6 veces mayor en el uso de CVC respecto a los pacientes con $F A V^{2,6-8}$.

Es por ello, que en el manejo de los catéteres venosos centrales en la práctica clínica cobran especial importancia los anticoagulantes utilizados en la permeabilización o el sellado del catéter ${ }^{4}$. En relación a ello, el fármaco de elección y de uso habitual en los servicios de hemodiálisis ha sido la heparina ${ }^{7}$, a pesar de que la literatura científica indica la existencia de una falta de consenso científico basado en la evidencia sobre el procedimiento de sellado y los volúmenes y las concentraciones de heparina-11. A ello se suma que el uso de este anticoagulante se asocia a una mayor incidencia de eventos adversos de trombocitopenia y hemorragia ${ }^{10,11}$.

En los últimos años, el uso del citrato se está presentando como una alternativa para el sellado de los ca- 
téteres venosos centrales, dado que tiene propiedades tanto anticoagulantes locales como antibmicrobianas, que a priori apuntan a una asunción de menores riesgos respecto al uso de la heparina ${ }^{3,9,11,12}$. De este modo, podría tratarse de una solución que además de cumplir con los requisitos de biocompatibilidad, baja toxicidad, actividad anticoagulante y acción antimicrobiana, puede garantizar menores riesgos para la seguridad del paciente. Por todo ello, a continuación se presenta una revisión y síntesis de la evidencia más actual y disponible existente en la literatura científi$\mathrm{ca}$, realizada con el fin de responder de forma comparada qué anticoagulante, la heparina o el citrato, presenta menos complicaciones de infección asociada al catéter venoso central en hemodiálisis.

\section{Material y Método}

El objetivo general de esta revisión bibliográfica es comparar qué anticoagulante, la heparina o el citrato, presenta menos complicaciones de infección asociada al catéter venoso central en hemodiálisis. Para responder a ello, se plantearon dos objetivos específicos: (1) identificar qué anticoagulante de uso en el sellado del catéter venoso central presenta menos complicaciones de bacteriemia asociada al catéter, y (2) identificar qué anticoagulante de uso en el sellado del catéter venoso central presenta menos complicaciones de colonización del catéter.

De este modo, se diseñó la estrategia de búsqueda bibliográfica siguiendo el principio de entropía, para acceder al mayor número de literatura científica disponible. A partir de las palabras clave "Catéter venoso central", "Infección asociada al catéter", "Heparina", "Citrato" y "Hemodiálisis" se identificaron los descriptores correspondientes en los tesauros DeCS y MeSH ("Catéteres Venosos Centrales"/"Central Venous Catheters", "Heparina"/"Heparin", "Citratos"/"Citrates", "Infecciones Relacionadas con Catéteres"/ "Catheter-Related Infections" y "Diálisis Renal"/"Renal Dialysis"). A continuación, se formularon dos operaciones booleanas, "Central Venous Catheters" AND ("Heparin" OR "Citrates") y "Central Venous Catheters" AND ("Heparin" OR "Citrates") AND ("Catheter-Related Infections" OR "Renal Dialysis"), y se consultaron el metabuscador EBSCOhost (Academic Search Premier, CINAHL) y las bases de datos PubMed, IME, Ibecs, LILACS, CUIDEN, Scopus y Cochrane Library. Entre los límites de búsqueda bibliográfica, se establecieron (1) publicación en los últimos 10 años (2005-2016), (2) idiomas castellano, inglés, portugués y francés, (3) estudios primarios cuantitativos y (4) estudios secundarios de revisión. Los resultados obtenidos fueron 276 artículos científicos de los que se seleccionaron finalmente 15 documentos para realizar la revisión bibliográfica, tras la eliminación de duplicados y la aplicación de criterios de inclusión y exclusión. Entre los criterios de inclusión se establecieron aquellos estudios con (1) uso de citrato o heparina, (2) determinación de la concentración de anticoagulante y (3) datos sobre IA-CVC, BA-CVC o colonización del catéter, mientras que entre los criterios de exclusión se establecieron aquellos estudios con (1) uso de anticoagulante en catéteres no definitivos, (2) pacientes con insuficiencia renal aguda, (3) muestra desadaptada al tamaño muestral y (4) déficit de calidad metodológica. (Tabla 1).

La mayoría de los resultados fueron estudios primarios realizados en Estados Unidos o Reino Unido, concretamente 5 ensayos clínicos y 5 cohortes. Los documentos restantes fueron estudios secundarios, 2 revisiones sistemáticas, 1 revisión sistemática con meta-análisis y 2 revisiones bibliográficas. En cuanto a los resultados objetivo, estos artículos científicos aportaron datos sobre IA-CVC, principalmente sobre bacteriemia evidenciada analítica y clínicamente, mientras que los resultados sobre colonización del catéter, o infección del punto de entrada, tuvieron menor disponibilidad en la literatura científica. También, se aportaron datos sobre trombosis, uso de terapia trombolítica, obstrucción, funcionalidad del catéter, flujo intradiálisis, KTV, ingreso hospitalario, recolocación del catéter o recambio del catéter, entre otros. Asimismo, las soluciones anticoagulantes utilizadas en estos estudios presentaron cierta variabilidad tanto en tipología como en concentración: heparina 5\%, heparina-gentamicina, citrato a baja concentración o $4 \%$, citrato a moderada concentración o $30 \%$ y citrato a alta concentración o 46,7\%, citrato-gentamicina, citrato-taurolidina y citrato-azul metileno-metilparabeno-propilparabeno. (Tabla 2). 
Tabla 1. Estrategia de búsqueda bibliográfica.

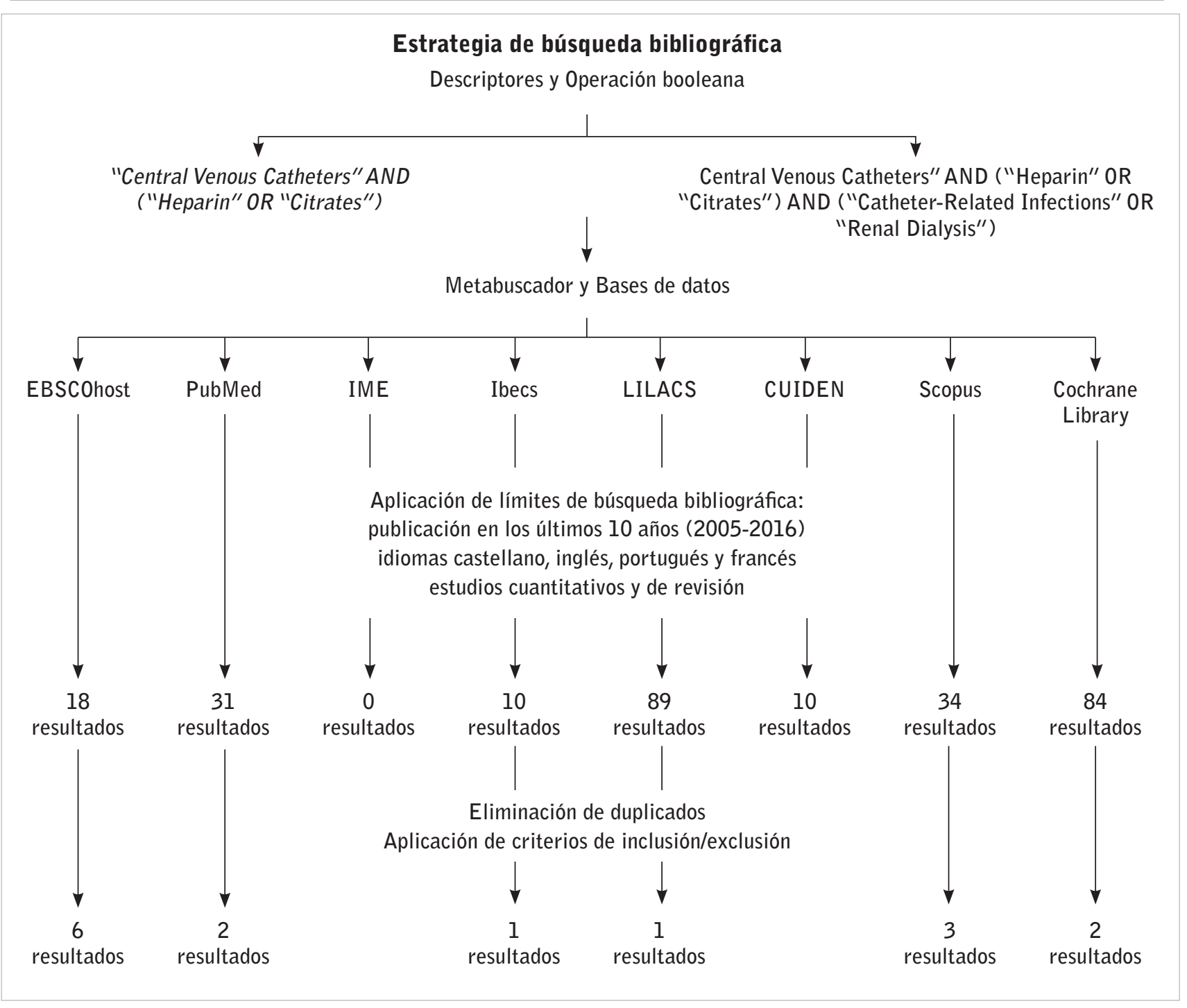

Tabla 2. Resultados y datos de la búsqueda bibliográfica.

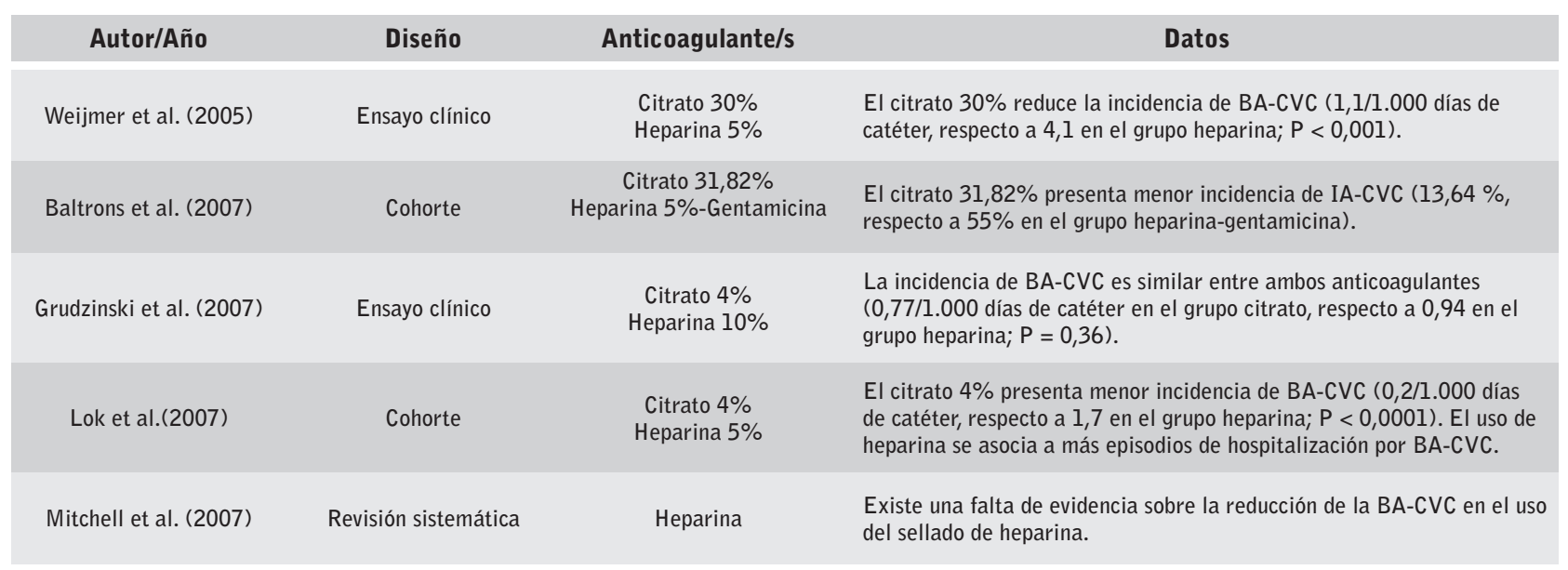


[ Mateu Nadal Servera, et al ]

En el sellado del catéter venoso central en hemodiálisis, ¿presenta el uso de citrato respecto al uso de heparina menos complicaciones...

\begin{tabular}{|c|c|c|c|}
\hline Autor/Año & Diseño & Anticoagulante/s & Datos \\
\hline Power et al. (2009) & Ensayo clínico & $\begin{array}{l}\text { Citrato } 47 \% \\
\text { Heparina } 5 \%\end{array}$ & $\begin{array}{l}\text { La incidencia de BA-CVC no difiere entre ambos anticoagulantes } \\
(0,7 / 1.000 \text { días de catéter). Tampoco se muestran diferencias entre } \\
\text { ambos anticoagulantes en la incidencia de colonización del catéter } \\
(0,7 / 1.000 \text { días de catéter en el grupo citrato, respecto a } 0,5 \text { en el grupo } \\
\text { heparina; } P=0,5) \text {. }\end{array}$ \\
\hline Venditto et al. (2010) & Cohorte & $\begin{array}{c}\text { Citrato } 46 \% \\
\text { Heparina } 5 \%-G e n t a m i c i n a \\
\text { Heparina } 5 \%\end{array}$ & $\begin{array}{l}\text { La heparina-gentamicina reduce la incidencia de IA-CVC (0,4/1.000 } \\
\text { días de catéter, respecto a } 2,9 \text { en el grupo heparina y } 3,4 \text { en el grupo } \\
\text { citrato; } P=0,06 \text { ). }\end{array}$ \\
\hline Bevilacqua et al. (2011) & Cohorte & $\begin{array}{l}\text { Citrato } 46,7 \% \\
\text { Heparina } 1,5 \%\end{array}$ & $\begin{array}{l}\text { El citrato } 46,7 \% \text { presenta menor incidencia de BA-CVC }(0,18 / 1.000 \\
\text { días de catéter, respecto a } 3,33 \text { en el grupo heparina; } P<0,001) \text {. }\end{array}$ \\
\hline Encarnaçao et al. (2013) & Revisión sistemática & Heparina & $\begin{array}{l}\text { Existe una falta de evidencia sobre la efectividad del sellado de heparina } \\
\text { en la reducción de la IA-CVC. }\end{array}$ \\
\hline Yon et al. (2013) & Ensayo clínico & $\begin{array}{l}\text { Citrato } 4 \% \\
\text { Heparina } 5 \%\end{array}$ & $\begin{array}{l}\text { El citrato } 4 \% \text { presenta menor incidencia de IA-CVC }(0,81 / 1.000 \text { días de } \\
\text { catéter, respecto a } 1,9 \text { en el grupo heparina; } P=0,026) \text {. }\end{array}$ \\
\hline Conway et al. (2014) & Revisión bibliográfica & Heparina & $\begin{array}{l}\text { Existe una falta de evidencia sobre las concentraciones y volúmenes en } \\
\text { el sellado de heparina, en relación con los eventos de IA-CVC. }\end{array}$ \\
\hline Moore et al. (2014) & Cohorte & $\begin{array}{l}\text { Citrato } 4 \% \text {-Gentamicina } \\
\quad \text { Heparina } 1 \%\end{array}$ & $\begin{array}{l}\text { El citrato } 4 \% \text {-gentamicina reduce en un } 73 \% \text { la incidencia de BA-CVC } \\
(0,45 / 1.000 \text { días de catéter, respecto a } 1,68 \text { en el grupo heparina; } P= \\
0,001 \text { ), con un menor riesgo de IA-CVC en relación con el sellado de } \\
\text { heparina (RR } 0,23 \text {; IC } 95 \% 0,13-038 \text { ). }\end{array}$ \\
\hline Zhao et al. (2014) & Meta-análisis & $\begin{array}{c}\text { Citrato } \\
\text { Citrato-gentamicina } \\
\text { Citrato-taurolidina } \\
\text { Citrato-azul metileno-metil- } \\
\text { parabeno-propilparabeno } \\
\text { Heparina }\end{array}$ & $\begin{array}{l}\text { El sellado de citrato reduce la incidencia de BA-CVC (RR } 0,39 ; \text { IC } 95 \% \\
0,27-0,56 ; \mathrm{P}<0,001 \text { ). El sellado de citrato asociado a antibiótico es } \\
\text { superior respecto a la heparina en la prevención de la } B A-C V C \text {, mientras } \\
\text { que el citrato exclusivo no presenta ventajas }(P<0,001, P=0,003, P= \\
0,008 \text { y } P=0,2 \text { respectivamente). }\end{array}$ \\
\hline Boersma et al. (2015) & Ensayo clínico & $\begin{array}{l}\text { Citrato } 46,7 \% \\
\text { Heparina } 5 \%\end{array}$ & $\begin{array}{l}\text { El citrato } 46,7 \% \text { presenta menor incidencia de BA-CVC ( } 31 \% \text {, respecto } \\
\text { a } 35 \% \text { en el grupo heparina). }\end{array}$ \\
\hline Hadaway et al. (2016) & Revisión bibliográfica & Heparina & $\begin{array}{l}\text { El sellado con heparina igual o inferior a } 500 \mathrm{UI} / \mathrm{mL} \text { reduce la inciden- } \\
\text { cia de IA-CVC, BA-CVC y colonización del catéter. }\end{array}$ \\
\hline
\end{tabular}

\section{Resultados y Discusión}

Los resultados más relevantes y actuales disponibles en la literatura científica presentaron cierta variabilidad, tanto en las concentraciones de anticoagulante como en la incidencia de infección, que dificulta concluir recomendaciones para la práctica clínica en el sellado de los catéteres venosos centrales en hemodiálisis. En líneas generales, por una parte se aporta numerosa evidencia sobre la BA-CVC, en relación con la concentración o combinación del anticoagulante de sellado, mientras que por otra parte los datos sobre colonización del catéter comparados entre el uso de citrato y heparina son más escasos.

\section{Bacteriemia asociada al catéter (BA-CVC)}

La bacteriemia relacionada con el catéter se define como el cuadro clínico de sepsis con foco aparente de infección en el dispositivo y aislamiento significativo del microorganismo (especie y antibiograma) tanto en hemocultivo extraído de vena periférica como en cultivo cuantitativo o semicuantitativo de la punta del catéter².

Entre los estudios revisados, uno de ellos un ensayo clínico aleatorizado que presenta resultados con significación estadística, se evidencia que la incidencia de BA-CVC es menor en el uso de citrato a concentraciones moderadas $(30 \%)$ y/o altas $(46,7 \%)$ respecto al uso de heparina al 5\%3,4,6. En este sentido, el uso de citrato $30 \%$ puede reducir el riesgo de BA-CVC en un $87 \%$ en catéteres tunelizados $(P<0,001)$ y un $64 \%$ 
en catéteres no tunelizados $(P=0,05)^{3}$. El ensayo clínico de Boersma et al. ${ }^{13}$, aunque realizado con pacientes que presentan además alteraciones hematológicas, añade que el citrato a altas concentraciones presenta una reducción estadísticamente significativa de la incidencia de infección asociada al catéter por bacterias gramnegativas, siendo 7 veces superior la tasa de BACVC en los casos de sellado con heparina y sin presentar diferencias en infecciones por grampositivas.

Sin embargo, estos resultados no coinciden con los expuestos en la revisión sistemática de Zhao et al..$^{5}$ y el ensayo clínico de Yon et al. ${ }^{12}$ en los que se indica que la incidencia de BA-CVC es menor en el uso de citrato a concentraciones bajas (4\%) y/o moderadas (30\%). En esta revisión sistemática, se concluye que el citrato a bajas ( $1-4 \%$ ) o moderadas ( $5-7 \%$ ) concentraciones se asocia a una disminución de la BA-CVC (RR 0,38, IC $95 \%, 0,25-0,58, P<0,00001$; $y$ RR 0,27, IC $95 \%$, $0,12-0,64, P=0,003$, respectivamente), siendo similar la incidencia de infección entre el citrato a altas concentraciones $(30-47 \%$ ) y la heparina (RR 0,50, IC $95 \%, 0,14-1,83, P=0,3)^{5}$. En el caso del citrato $4 \%$, se asocia con significación estadística a una menor incidencia de BA-CVC, presentándose como un anticoagulante eficiente que reduce además los costes sanitarios por ingreso hospitalario, recolocación del catéter y recambio del catéter ${ }^{12}$. Por otra parte, en dos ensayos clínicos y dos revisiones sistemáticas, se evidencia que la incidencia BA-CVC es similar en el uso de heparina respecto al uso de citrato tanto a altas ${ }^{11,14}$ como a bajas ${ }^{9}$ concentraciones.

En cuanto a la combinación del anticoagulante, la literatura científica aporta cada vez más resultados sobre la asociación de heparina o citrato con antibióticos. El uso comparado de citrato y heparina gentamicina no muestra resultados coincidentes entre los estudios revisados, puesto que mientras que el estudio de Baltrons et al. ${ }^{4}$ afirma que la incidencia de IACVC es del $13,64 \%$ en el sellado con citrato $31,82 \%$ respecto al $55 \%$ en el sellado con heparina-gentamicina, los resultados de Venditto et al. ${ }^{14}$ concluyen que la heparina-gentamicina reduce la incidencia de IA-CVC $(0,4 / 1.000$ días de catéter, respecto a 2,9 en el grupo heparina y 3,4 en el grupo citrato; $\mathrm{P}=$ $0,06)$. Por otra parte, la revisión sistemática con meta-análisis de Zhao et al. ${ }^{5}$ concluye en el análisis de subgrupos que el citrato asociado a antimicrobianos es más efectivo en la prevención de la BA-CVC respecto a la heparina sódica o el citrato exclusivo (citrato-gentamicina, RR 0,25, IC 95\%, 0,13-0,47, P < 0,001; citrato-taurolidina, RR 0,45, IC 95\%, 0,27-
$0,77, P=0,003$; y citrato-azul metileno-metilparabeno-propilparabeno, RR 0,25, IC 95\%, 0,13-0,47, $\mathrm{P}<0,001)$.

\section{Colonización del catéter (infección del punto de entrada)}

La colonización del catéter se define como el resultado de aislamiento significativo del microorganismo en punta de catéter o en puntos de conexión a partir de cultivo cuantitativo o semicuantitativo, pudiendo ser clínicamente documentada con signos locales de infección (dolor, rubor, calor, enduración, pus) y sin bacteriemia ${ }^{2}$.

A pesar de que la evidencia existente sobre la infección del punto de entrada en CVC es menor y poco concluyente, además de la menor relevancia respecto a la BA-CVC, la revisión sistemática de Zhao et al. ${ }^{5} \mathrm{y}$ el ensayo clínico de Power et al. ${ }^{11}$ añaden que tampoco existen diferencias significativas en la incidencia de formación de biofilm y colonización del catéter. De hecho, existe una falta de evidencia sobre las concentraciones y volúmenes en el sellado de heparina $^{7}$ que permita comparar resultados y establecer indicaciones claras. Sin embargo, mientras que en un estudio de cohortes comparativo se indica que la heparina estimula la formación de biofilm y se asocia a una mayor incidencia de colonización del catéter en relación con el sellado de citrato ${ }^{8}$, una revisión bibliográfica de diversos ensayos clínicos sobre el uso de heparina en la permeabilización de los catéteres venosos centrales concluye que el uso de concentraciones de heparina inferiores al $5 \%$ o $500 \mathrm{UI} / \mathrm{mL}$ no se asocia a un incremento de la incidencia de infección del punto de entrada ${ }^{15}$.

Por tanto, existe una contrariedad en los resultados sobre las complicaciones de infección asociada al catéter, tanto de BA-CVC como de colonización del catéter. Aun así, se puede describir que la literatura científica se centra cada vez más en la combinación de estos anticoagulantes con antibióticos tipo gentamicina, a pesar de que la resistencia antibiótica es un problema a estudio ${ }^{8,14}$. También, es necesario tener en cuenta otros factores relacionados como los casos de hemorragia, trombocitopenia, trombosis $u$ obstrucción $n^{7,10,16,17}$, además de la relación coste-efectividad ${ }^{9}$, por lo que a las recomendaciones sistemáticas debe sumarse la individualización del uso de un anticoagulante u otro, en función de los parámetros hidroelectrolíticos y hematológicos y los antecedentes de funcionalidad e infección del catéter. 


\section{Conclusiones}

La heparina ha sido aceptada como el anticoagulante estándar para el sellado de los catéteres venosos centrales, estando el citrato reservado a aquellos pacientes que presentan alergia a la heparina o trombocitopenia, entre otras causas. La alta variabilidad en los resultados, tanto en la IA-CVC, la BA-CVC y la colonización del catéter como en la aplicación de concentraciones y volúmenes, a priori no establece indicaciones concluyentes sobre el uso prioritario del citrato o la heparina de forma sistemática en la práctica clínica en hemodiálisis.

Sin restar importancia al sellado del catéter venoso central en hemodiálisis, propósito de este estudio, el uso de un anticoagulante u otro podría tener menor importancia, requiriéndose fundamentalmente para la prevención de la IA-CVC unas medidas de esterilidad en la cura del punto de entrada del catéter y de asepsia en el manejo de las luces y los orificios de éste. Sin embargo, esta revisión de la literatura permite apuntar que aunque el uso de citrato sódico exclusivo no presenta claras ventajas en las complicaciones de IA-CVC respecto a la heparina sódica, sí parecen demostrados sus beneficios a bajas (4\%) o moderadas $(30 \%)$ concentraciones, y especialmente, al combinarlo con antibióticos. Aún así, la asociación del citrato con antibióticos debe ser estudiada con profundidad, especialmente por la comorbilidad del paciente en hemodiálisis, la reincidencia de IA-CVC y la resistencia antibiótica. En este sentido, son necesarios más estudios tipo ensayo clínico o cohorte en los que el uso de concentraciones y volúmenes de anticoagulante sean comparables para poder discutir y concluir qué recomendaciones deben ser transferidas en la práctica clínica en hemodiálisis.

\begin{tabular}{|c|}
\hline Recibido: 16 julio 2016 \\
Revisado: 1 diciembre 2016 \\
Modificado: 12 diciembre 2016 \\
Aceptado: 3 febrero 2017 \\
\hline
\end{tabular}

\section{Bibliografía}

1. Rodríguez Hernández JA, González Parra E, Julián Gutiérrez JM, Segarra Medrano A, Almirante B, Martínez MT, et al. Guías de acceso vascular en hemodiálisis. Rev Nefrol [Internet]. 2005;25(1):397.

2. Fariñas MC, García-Palomo JD, Gutiérrez-Cuadra M. Infecciones asociadas a los catéteres para hemodiálisis y diálisis peritoneal. Enferm Infecc Microbiol Clin [Internet]. 2008;26(8):518-26.

3. Weijmer $M C$, van den Dorpel $M A$, Van de Ven PJG, ter Wee PM, van Geelen JACA, Groeneveld $\mathrm{J} 0$, et al. Randomized, clinical trial comparison of trisodium citrate $30 \%$ and heparin as catheterlocking solution in hemodialysis patients. J Am Soc Nephrol [Internet]. 2005 Sep;16(9):2769-77.

4. Baltrons Bosch A, Coll Vinyoles $S$, Font Senen C, Jubany López M, Plana Illa C, Sunyer García A. Estudio comparativo del sellado de catéteres con citrato trisódico o heparina sódica más gentamicina. Rev Soc Esp Enferm Nefrol [Internet]. 2007;11(2):19-24.

5. Zhao Y, Li Z, Zhang L, Yang J, Yang Y, Tang Y, et al. Citrate versus heparin lock for hemodialysis catheters: a systematic review and meta-analysis of randomized controlled trials. Am J Kidney Dis [Internet]. 2014 Mar;63(3):479-90.

6. Bevilacqua JL, Gomes JG, Santos VFB, Canziani MEF. Comparação entre citrato trissódico e heparina como solução para selo de catéter em pacientes em hemodiálise. J Bras Nefrol [Internet]. 2011 Mar;33(1):86-92.

7. Conway MA, McCollom C, Bannon C. Central Venous Catheter Flushing Recommendations: A Systematic Evidence-Based Practice Review. J Pediatr Oncol Nurs [Internet]. $2014 ; 31(4): 185-$ 90.

8. Moore CL, Besarab A, Ajluni M, Soi V, Peterson EL, Johnson LE, et al. Comparative effectiveness of two catheter locking solutions to reduce catheterrelated bloodstream infection in hemodialysis patients. Clin J Am Soc Nephrol [Internet]. 2014 Jul;9(7):1232-9. 
9. Grudzinski L, Quinan P, Kwok S, Pierratos A. Sodium citrate $4 \%$ locking solution for central venous dialysis catheters - an effective, more cost-efficient alternative to heparin. Nephrol Dial Transplant [Internet]. 2007 Feb;22(2):471-6.

10. Mitchell MD, Anderson BJ, Williams K, Umscheid CA. Heparin flushing and other interventions to maintain patency of central venous catheters: a systematic review. J Adv Nurs [Internet]. 2009 0ct;65(10):2007-21.

11. Power A, Duncan N, Singh SK, Brown W, Dalby E, Edwards $C$, et al. Sodium citrate versus heparin catheter locks for cuffed central venous catheters: a single-center randomized controlled trial. $\mathrm{Am} \mathrm{J}$ Kidney Dis [Internet]. 2009 Jun;53(6):1034-41.

12. Yon CK, Low CL. Sodium citrate $4 \%$ versus heparin as a lock solution in hemodialysis patients with central venous catheters. Am J Heal Pharm [Internet]. 2013 Jan 15;70(2):131-6.

13. Boersma RS, Jie KS, Voogd AC, Hamulyak K, Verbon A, Schouten HC. Concentrated citrate locking in order to reduce the long-term complications of central venous catheters: a randomized controlled trial in patients with hematological malignancies. Support Care Cancer [Internet]. 2015 Jan;23(1):37-45.
14. Venditto $M$, du Montcel ST, Robert J, Trystam D, Dighiero J, Hue D, et al. Effect of catheterlock solutions on catheter-related infection and inflammatory syndrome in hemodialysis patients: heparin versus citrate $46 \%$ versus heparin/gentamicin. Blood Purif [Internet]. 2010 Jan;29(3):268-73.

15. Hadaway L. Heparin Locking for Central Venous Catheters. J Assoc Vasc Access [Internet]. 2006 Jan;11(4):224-31.

16. Lok CE, Appleton D, Bhola C, Khoo B, Richardson RMA. Trisodium citrate $4 \%$ - an alternative to heparin capping of haemodialysis catheters. Nephrol Dial Transplant [Internet]. 2007 Mar;22(2):477-83.

17. Encarnação R, Marques P. Permeabilidade do cateter venoso central: uma revisão sistemática da literatura. Rev Enferm Ref [Internet]. 2013 Mar 29;3(9):161-9. 